ARTICLE OPEN

\title{
3D printed leech-inspired origami dry electrodes for electrophysiology sensing robots
}

\author{
Tae-Ho Kim ${ }^{1}$, Chao Bao ${ }^{1}$, Ziniu Chen ${ }^{1}$ and Woo Soo Kim (iD ${ }^{1 凶}$
}

In this study, based on inspiration drawn from origami and the suction mechanism of leeches, a dry electrode is developed for reliable blood pressure (BP) monitoring. The leech-inspired suction mechanism generated a local soft vacuum facilitating appropriate contact with the human skin. Subsequently, an electrocardiogram (ECG) sensor, termed a leech-inspired origami (LIO) sensor, was constructed using the developed dry electrode. The LIO with a sensing robot system ensures reliable ECG signals with a signal-to-noise ratio of $21.7 \pm 0.56 \mathrm{~dB}$. From the paired detection of ECG and photoplethysmography (PPG) through human-robot interaction, BP monitoring was demonstrated. The average difference of the systolic BP between that estimated by the sensing robot and that monitored by the sphygmomanometer was $0.03 \mathrm{mmHg}$, indicating the reliable BP monitoring ability of the sensing robot. The LIO sensing system inspired by origami and leech behaviors makes BP sensing tools feasible, which in turn would further the development of a remote healthcare monitoring robotic system.

npj Flexible Electronics (2022)6:5; https://doi.org/10.1038/s41528-022-00139-x

\section{INTRODUCTION}

Remote monitoring systems of human healthcare have been widely studied because they can reduce the risk of secondary infection among healthcare workers ${ }^{1}$. Robotics offers a promising method to mitigate risk and improve patient care effectiveness and quality as a focused remote healthcare technology ${ }^{2}$. Blood pressure (BP) is an essential medical diagnostic tool in the medical healthcare system because high BP is closely related to several chronic diseases ${ }^{3}$. In addition, hypertension or high BP is a preexisting symptom or precursor of possible infection in patients. This increases the demand for BP monitoring systems of patients in their daily lives ${ }^{4}$. From this point of view, the use of sensing robots in medical healthcare systems has substantial advantages because they can assist healthcare workers in monitoring the vital signs of patients and creating a friendly environment for those patients who may need to be isolated ${ }^{3}$. For the development of BP monitoring using robots, conventional methods with a cuff-based sphygmomanometer are not feasible because they cannot perform continuous monitoring of patients ${ }^{4}$. Thus, a cuffless monitoring system combining sensing tools of electrocardiogram $(E C G)^{5}$ and photoplethysmography (PPG) ${ }^{6}$ is rational. The pulse arrival time (PAT) parameter, which indicates the time taken for the pulse waveforms to traverse from the heart to a distal site, is often used in a paired sensing system ${ }^{7}$. PAT is determined using fiducial points, including the R-wave and the systolic peak of the ECG and PPG waveforms ${ }^{8,9}$. In addition, unlike a sphygmomanometer, an ECG-PPG paired sensing system can continuously monitor $\mathrm{BP}^{8,10}$. Moreover, once such healthcare tools are integrated with sensing robot systems, they can form a bridge in remote or general monitoring between medical personnel and patients receiving outpatient care.

However, an ECG sensing system, an essential component for BP monitoring, requires direct contact with the human skin to collect the cardiac signals; moreover, there is the issue of high noise signals depending on the contact conditions ${ }^{11}$. Therefore, wet- or stickytype electrodes are employed for the system ${ }^{12}$. Although such wettype electrodes are appropriate for solving the contact issue, they can cause skin irritation and allergy in patients ${ }^{13,14}$. In addition, it is not appropriate to monitor BP with the robot because, at each measurement, the electrodes need to be replaced or treated. Consequently, the demands for dry electrodes for ECG sensors have dramatically increased, reducing the risk of skin troubles and requiring less treatment during $\mathrm{BP}$ measurement ${ }^{15}$. However, the use of dry electrodes for the ECG sensor causes contact drawbacks ${ }^{16}$. Hence, methods to impose adhesive properties to dry electrodes for ECG sensors are urgently required. In recent years, conducting polymers such as poly (3,4-ethylene dioxythiophene): polystyrene sulfonate (PEDOT: PSS) have been used as the dry electrode of the ECG sensor after inserting silver/silver chloride $(\mathrm{Ag} / \mathrm{AgCl})$ into the conducting polymer matrix to improve the interface with the skin ${ }^{17}$. In addition, self-adhesive electrodes for the sensor were designed using extra soft or microstructurally patterned polymers ${ }^{18-23}$. Moreover, printing patterned dry electrodes on stretchable polymers has been reported to improve adhesion to the $\operatorname{skin}^{24}$. Although the aforementioned studies contribute to improving the inadequacies of dry electrodes of ECG sensors, additional support systems such as belts- or tapes-tightening to secure the adhesion of the dry electrodes to the skin are still required, which can make frequent monitoring challenging and inconvenience of the patients.

A simplified compact hardware of the system, which encompasses all the aforementioned requirements, is required ${ }^{14}$. Otherwise, the sensing system would hinder actual robot motion. Therefore, among possible solutions, the architectural properties of origami are strongly considered because origami has peculiar mechanical properties derived from decorative folding patterns ${ }^{25}$. For example, the mechanical rigidity of the structure is tunable by the customization of folding patterns on the structure. In addition, origami has force direction-shifting properties such as rotating ${ }^{26}$ or bending ${ }^{27}$ using a simple axial force. Moreover, the physical dynamics of origami mainly occur on the crease line such that the patterned planes or facets are scarcely affected, which can be utilized as potential location sites of sensing units ${ }^{28}$.

Origami has many folding patterns and methods for generating various types of $3 \mathrm{D}$ structures. Also, origami has shown unique

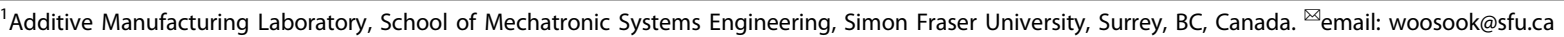




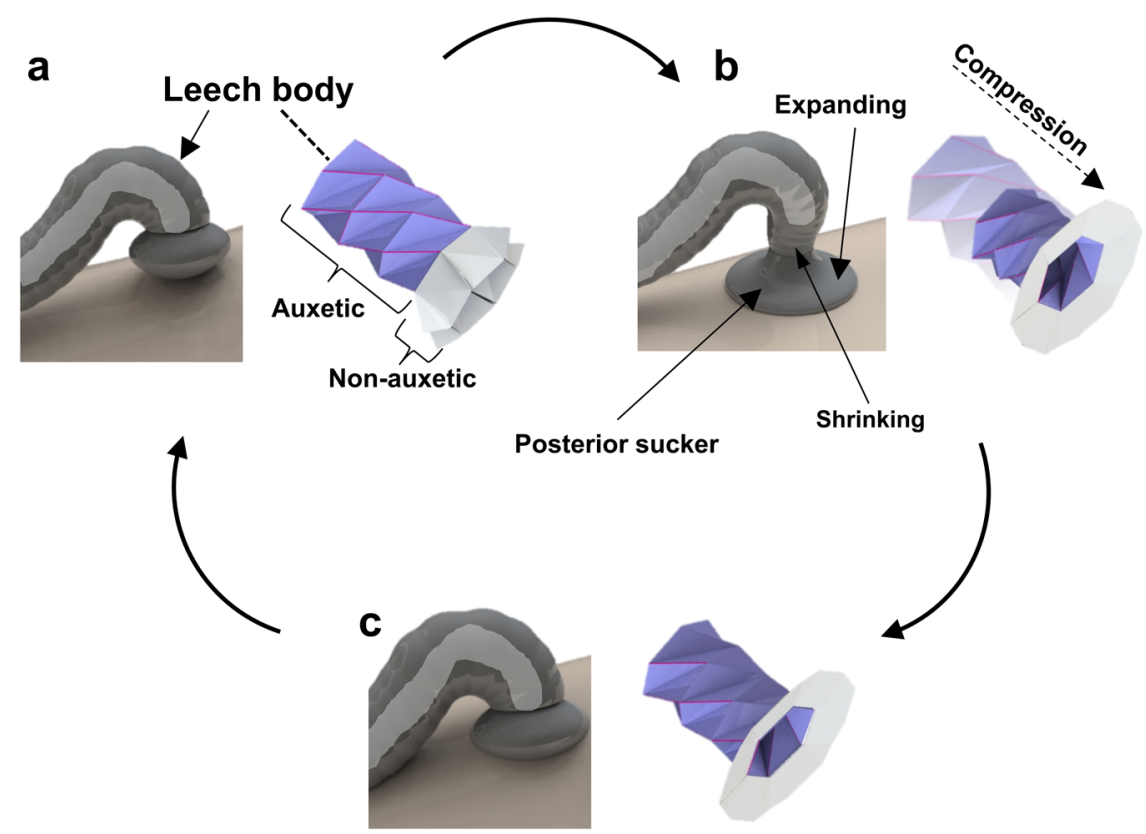

Fig. 1 Design of Leech-inspired origami (LIO). Schematics of the sticking mechanism of the posterior leech sucker. a Before the attachment, the shrunk sucker is positioned to target the skin surface. b The sucker part of the leech is stretched out along the radial direction and shrunken the body to attach to the skin. c The leech's body expands for the pumping to the victim's blood. The body trunk of the leech was mimicked by auxetic design, and the sucker was mimicked by non-auxetic origami design. The 3D origami composed of non-auxetic tip and the auxetic trunk is proposed in this study.

characteristics such as suction for grasping ${ }^{29}$, and foldability inspired by nature, such as the origami robot inspired by ladybug wings or dual morphing pelican eels ${ }^{30,31}$. Among complex mechanisms of leech's adhesive feature, it has an expandable posterior sucker and body ${ }^{32}$. Its organs expand and shrink appropriately to maintain better adhesion to the victim ${ }^{33,34}$. This adhering mechanism can be considered as the simultaneous motion of non ${ }^{35}$ and auxetic ${ }^{36}$ structures. From this point of view, origami can achieve such motion in a monolithic structure using folding patterns ${ }^{26}$. Moreover, tuning or customizing processes are available after the development of such nature-inspired origami ${ }^{26}$.

In this study, a leech-inspired origami (LIO) sensor was developed to monitor biomedical vital signs using a humanoidsensing robot. The difficulty in continuing essential health services during the COVID-19 pandemics provides a lesson on the needs of remote health care systems. Thus, a robot-assisted system for BP monitoring is an ideal remote healthcare system. Dry serpentine electrodes were three-dimensionally (3D) printed on the $\mathrm{LIO}$ sensor for their stability against the expansion and contraction of the LIO sensor. The developed LIO fingertip sensors were integrated into the humanoid robot and demonstrated reliable ECG sensing performance compared to commercial wet ECG electrodes. Finally, a BP monitoring robot system composed of paired ECG and PPG sensing hands was demonstrated by human-robot interaction. Different BP profiles were obtained from the developed sensing robot system through interaction with human subjects under different exercise conditions.

\section{RESULTS AND DISCUSSION}

\section{Design of the LIO sensor}

Leeches expand the mouth (sucker) to increase the contact area with the skin, and simultaneously, its body is shrunk to prepare for the pumping activity to suck the blood of the victim, as presented in Fig. 1a, $b^{32}$. During sucking, its expanded mouth is shrunk, and the body is expanded, as shown in Fig. 1c. Structurally, such a mechanism is considered a combination of simultaneous non-auxetic and auxetic behaviors. Based on this mechanism, an origami composed of a non-auxetic tip with auxetic bodies was developed (Fig. 2a). Once the non-auxetic structure in the origami is expanded, the auxetic part shrinks under compression, as shown in Fig. 2b. During compression, the area of the non-auxetic part in origami increases as observed in Fig. $2 c$ in the $x, y$ plane view, implying the expansion of the nonauxetic part in origami. By contrast, the auxetic part is shrunk, indicating a reduction in the inner space. In addition, the LIO has distinctive features, including rotating when a $z$-directional force is applied. The details of the structural behaviors of the origami are presented in Supplementary Video 1. The corresponding area changes were measured depending on the compressed heights in Fig. $2 d$. The area of the non-auxetic structure shows linearly upward trends at a more compressed length, $\Delta h$, and at $8 \mathrm{~mm}$ of the length, its area at the $x, y$ plane view is 1.6 times larger than the de-compressed state. In the auxetic origami part, the area is reduced to a factor of 0.4 after compression. The rotating behavior of the origami structure is shown in Fig. 2e. The rotating angle of the origami has a linear relationship with $\Delta h$, indicating that the force is converted from the $z$-directional force to torque. With these structural features, the origami structure is expected to form a standby state to pump after compression; the structure starts sucking during decompression like a leech. As shown in Fig. $2 \mathrm{f}$ and Supplementary Video 2, the LIO stably contacts and sucks the human finger such that the LIO is still attached to the fingertip when the finger is moved.

\section{Parametric study to optimize the LIO}

As mentioned earlier, origami can generate various 3D structures, even mimicking bio-nature. Beyond structural generation, its structure can be customized to improve specific mechanical properties. For the optimized conformal contact of origami for the development of the ECG sensing unit, the LIO is parametrically studied with tunable factors. The auxetic part of the LIO is composed of a three-layered Kresling origami structure ${ }^{27}$. Detailed information about the Kresling origami is described in Supplementary Note 1 

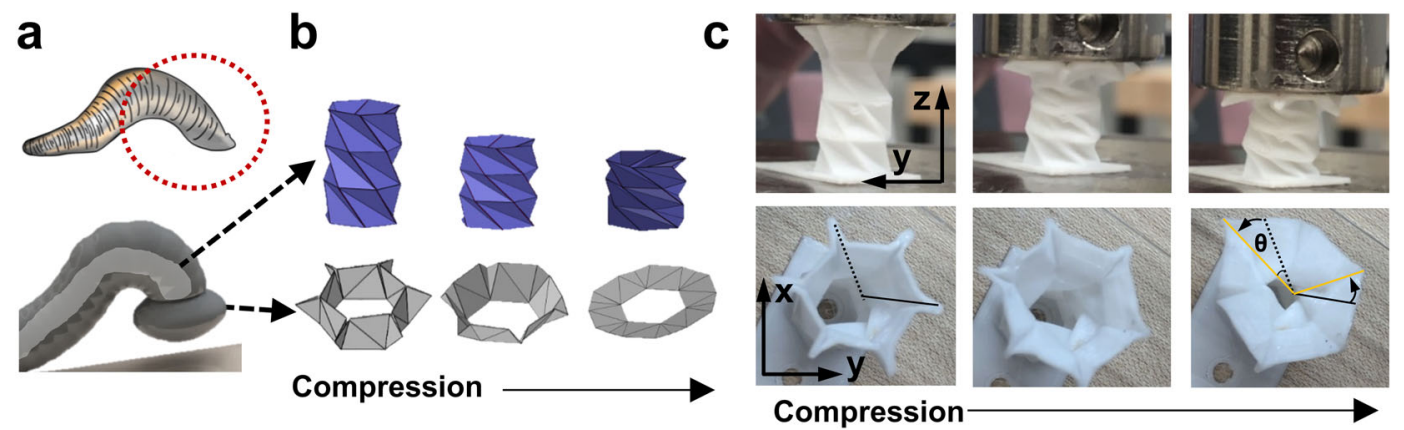

d
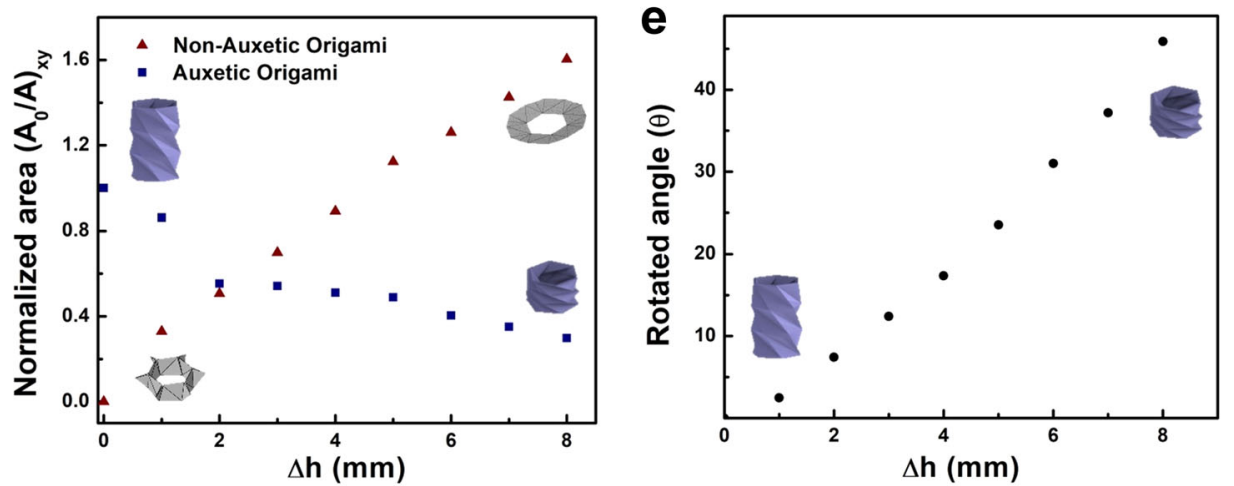

f
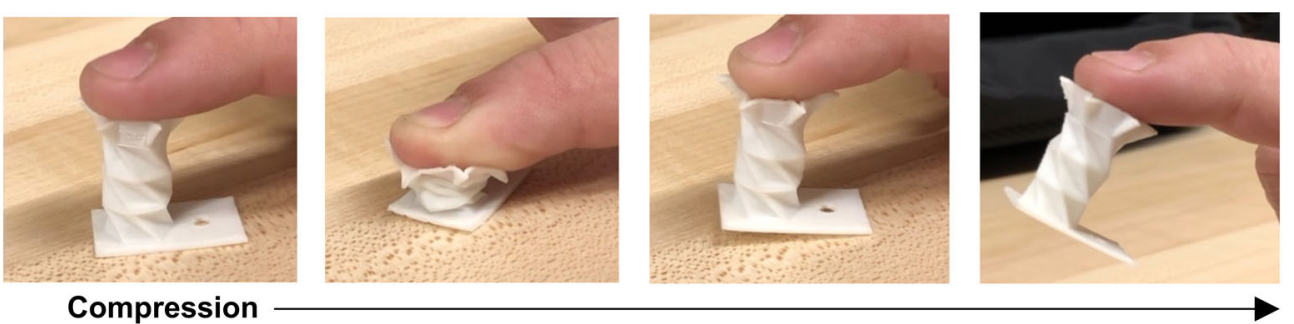

Fig. 2 The mechanical behavior of the designed LIO structure. a Schematics of the posterior sucker and body of leech and $\mathbf{b}$ their corresponding leech-inspired non- and auxetic origamis with the folding features under compression. c The actual images of 3D printed LIO under compression, side views (top), and top views (bottom). d The normalized area changes of LIO. e Rotated angles depending on compressed distances. $\mathbf{f}$ Representative photos of the adhesive performance of the designed LIO.

and Supplementary Fig. 1. When angular designs of triangular patterns are tailored on origami structures, as shown in Fig. 3a, the mechanical properties of the structure are adaptable. In this parametric study, we compared the responses of three different parameters at different angles: (1) Poisson's ratio, (2) rotation angle, and (3) elastic modulus of the origami structures ${ }^{26,27}$.

Poisson's ratio reflects the degree of perpendicular strain depending on the axial force. Thus, when the Poisson's ratio is changed, the volume ratio before and after compression is varied. Consequently, the volume ratio affects the suction strength of the origami structure by generating a vacuum. Such a vacuum is estimated using Eqs. 1 and 2.

Vacuum $\%: P_{\mathrm{v} \%}=100 \%-\left(P_{o} / P_{\mathrm{atm}}\right) \times 100 \%$

Ideal gas equation : $P_{\mathrm{o}}=n R T / V_{\mathrm{o}}$

where $P_{\mathrm{v} \%}, P_{\mathrm{o}}, P_{\mathrm{atm}}$, and $V_{\mathrm{o}}$ are the percent of vacuum (vacuum \%), the absolute pressure in the origami structure, absolute pressure in the standard atmosphere, and the actual inner volume of the structure, respectively. It can be seen that $P_{\mathrm{v} \%}$ is proportional to $V_{\mathrm{o}}$. Thus, the more negative the Poisson's ratio of the origami structure, the higher the suction strength. Figure $3 \mathrm{~b}$ shows the Poisson's ratio profiles at different $z$-directional strains ( $\varepsilon_{\text {axial }}$ and axial strain) with various angular designs on origami. The transverse strain $\left(\varepsilon_{\text {trans }}\right)$ is represented by the blue line in Fig. $3 c$. The lowest Poisson's ratios with negative values are observed from the auxetic part at a strain of approximately 0.15 , indicating a fully shrunken state in the transverse direction. By contrast, the nonauxetic structure shows the highest Poisson's ratio of approximately 1.7 , with positive values at a strain of approximately 0.25 . At a fixed a angle, a higher $\beta$ angular design shows a lower Poisson's ratio. The boundary conditions for the $a$ and $\beta$ angles are described in Supplementary Note 1 with Supplementary Fig. 2.

Additionally, we consider that the rotating behavior of the origami structure is an important property. The rotating of origami under compression results in more effective contact with the human skin because of its rubbing effects. Thus, it can be concluded that the rotating feature of origami needs to be investigated for better ECG sensing performance. The rotation angles of the origami structure are shown in Fig. 3d. They also exhibited linear trends at higher angular designs of the auxetic parts. However, the non-auxetic structure is nonrotational with the $z$-directional force. Thus, the rotation of the entire structure is primarily caused by auxetic elements.

These two physical properties of LIO are derived from the collapsing or folding behavior, which is affected by the rigidity of the origami creases, as presented in Supplementary Fig. 1a, b. Thus, the structural rigidity of the origami was investigated. Compression tests of the origami were performed to obtain the elastic modulus (Fig. 3e). The stress-strain curve has three distinctive peaks at approximately 15,30 , and $45 \%$ strains, derived from the multi-stability of the origami ${ }^{26,27}$. The three different elastic moduli were measured from the various angular origami designs shown in Fig. 3f. Generally, the higher $\beta$ angular designs 
a

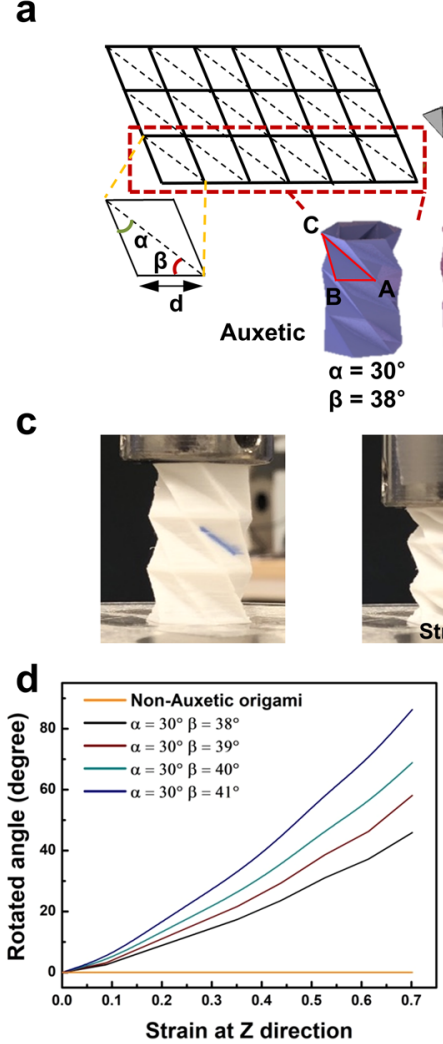

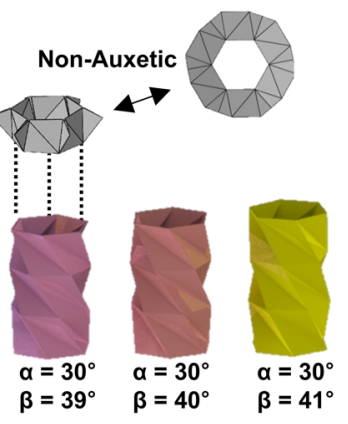

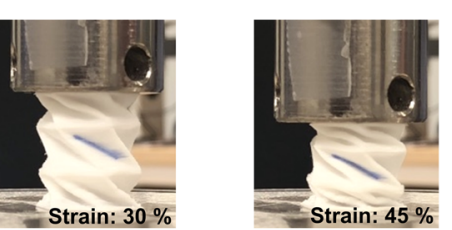

b
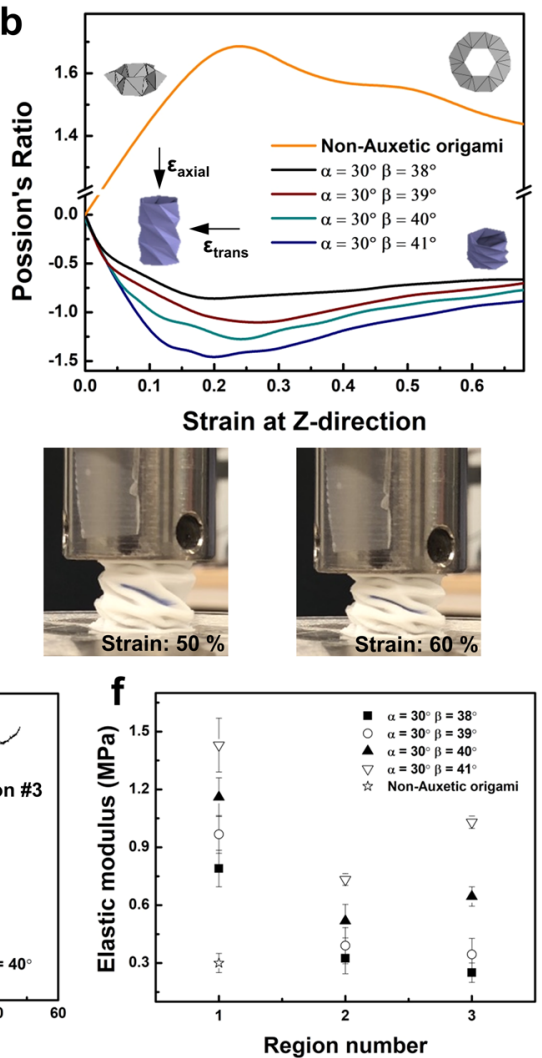

Fig. 3 Parametric study for the optimization of LIO. a Schematics of different leech-inspired origami (LIO) designs. The auxetic origami is tailored by changing the angle of triangular patterns. b Poisson's ratio of the non-auxetic and auxetic parts of the LIO at the $z$-directional strain. c Actual images of auxetic origami to represent transverse strain $\left(\varepsilon_{\text {trans }}\right)$ at $z$-directional compression $\left(\varepsilon_{\text {axial }}\right)$. $\mathbf{d}$ Rotated angles at the $z$-directional compression. e The stress-stain curve of the LIO design with the angular of $\sigma=30^{\circ}, \beta=40^{\circ}$. $f$ Elastic modulus of LIO designs with different angles. Error bars represent the standard deviation.

of the LIO structure show a greater elastic modulus. The structure with $\beta=38^{\circ}$ has $0.79 \pm 0.090,0.33 \pm 0.081$, and $0.25 \pm 0.054 \mathrm{MPa}$ for regions \#1 to \#3, respectively, as the lowest values among the auxetic samples. At $\beta=39^{\circ}$, elastic moduli of $0.98 \pm 0.096,0.39 \pm$ 0.082 , and $0.35 \pm 0.078 \mathrm{MPa}$ are obtained for regions \#1 to \#3, respectively. The elastic modulus of region $\# 1$ for both designs is more than twice as high as that of regions \#2 and \#3, owing to the force-dispersing properties of the origami structure or structural rigidity before the collapse initiation ${ }^{26,37}$. After the first region, the LIO structure gradually collapses during compression, as shown in Supplementary Videos 1 and 2, reflecting a similar elastic modulus at regions \#2 and \#3. In the case of the angular designs, at $\beta=$ $40^{\circ}$, the elastic modulus of region \#2 $(0.52 \pm 0.086 \mathrm{MPa})$ is lower than that of the other regions ( $\# 3,0.65 \pm 0.050 \mathrm{MPa})$. At $\beta=41^{\circ}$, the difference $\left(\Delta E=E_{\# 3}-E_{\# 2}\right)$ of the elastic modulus between regions \#2 and \#3, $0.73 \pm 0.031 \mathrm{MPa}$, and $1.03 \pm 0.032 \mathrm{MPa}$, respectively, is $0.30 \mathrm{MPa}$, which is higher than $0.13 \mathrm{MPa}$ at $\beta=$ $40^{\circ}$. This finding implies that the LIO structure with an angular design of $\beta>40^{\circ}$ does not gradually collapse; the partial collapse of the 2nd origami layer occurs with the 1st layer. This finding implies that the structural rigidity is close to the material rigidity assumed from the stress-strain curve beyond region \#3 after the modification of the angular designs of the $\mathrm{LIO}$ of $\beta>40^{\circ}$. LIO with $\beta=42^{\circ}$ does not show collapsing or folding behaviors, as shown in Supplementary Fig. $2 \mathrm{~b}$, because the structural rigidity of the LIO is greater than its material property. Based on the parametric study, an origami structure with $a=30^{\circ}$ and $\beta=40^{\circ}$ was selected as the optimal candidate for the LIO of the conformal ECG sensor. As the optimal criteria, we considered the angular designs for the maximum Poisson's ratio and rotation angles of the LIO structure; however, to maintain the better folding behavior of the LIO, an angular design with $\beta=40^{\circ}$ was selected instead of $\beta=41^{\circ}$. Adhesion force has been measured by using the pull-off force test, as shown in Supplementary Fig. 3c. The LIO with $a=30^{\circ}$ and $\beta=$ $40^{\circ}$ shows $1.21 \pm 0.24 \mathrm{~N}$.

\section{Conductive serpentine for ECG electrode design}

In the design of reliable ECG electrodes, the electrode must be robust against the strain when it is expanded. The expansion of the LIO structure is a result of the combination of both $x$-and $y$-directional deformations. Although the origami is mainly deformed at the crease line without theoretical plane deformation, an apparent strain or stress distribution exists on the plane in actual cases $^{38}$. Thus, the design of a stable structure for ECG electrodes with planar deformation is required. Serpentine-patterned electrodes were printed on the non-auxetic plane of the LIO. As demonstrated in Fig. 4a, the non-auxetic part of the LIO is fixed on the body of the patient after expansion. The serpentine design is composed of four different semi-circular lines in a repeatable pattern known as reliable stretchability against planar deformation ${ }^{25}$.

In addition, full integration along the non-auxetic part and proper contact of the skin with the printed electrodes need to be secured. Therefore, the serpentine electrodes at the valley of the LIO are connected using a free-standing serpentine path, as shown at the bottom right of Fig. $4 \mathrm{~b}$. The distance $(d)$ of the valley at the non-auxetic part increases when the LIO is in contact with the skin. The force direction of $d$ has two directional elements; the length of $d$ is proportionally varied at compressed $\Delta h$, as shown in Fig. 4c. At the fully expanded state of the non-auxetic part of the LIO, strain $d$ reaches approximately 1.8 . In Fig. $4 d$, the printed serpentine electrodes with a number of $n=1-3$ layers are 
a

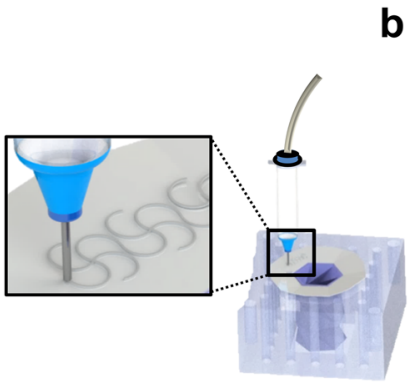

b

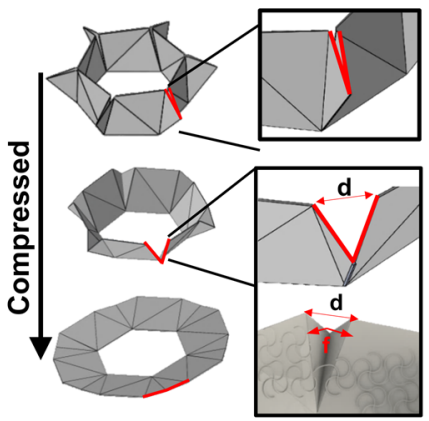

d
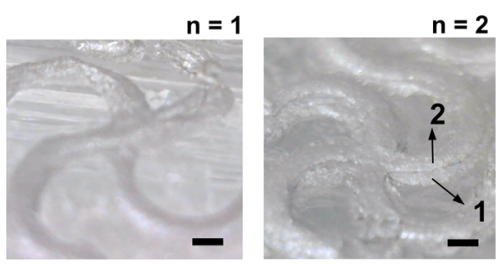

f

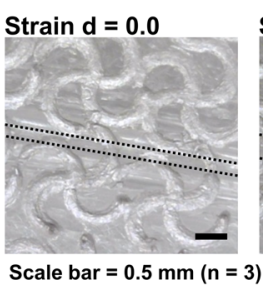

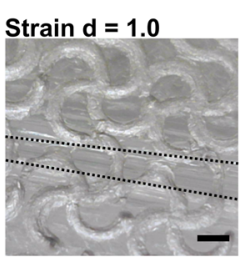

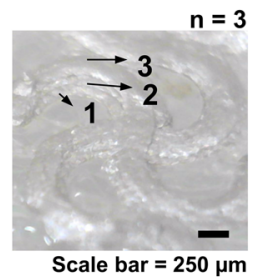

Strain $d=1.8$

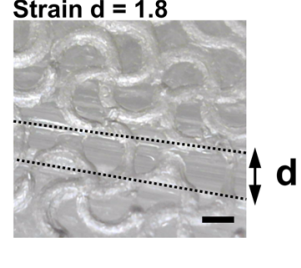

C

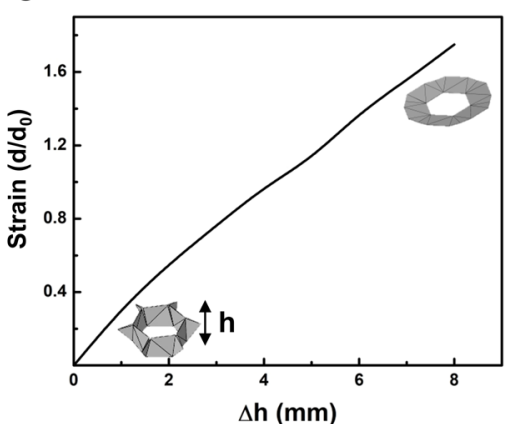

e

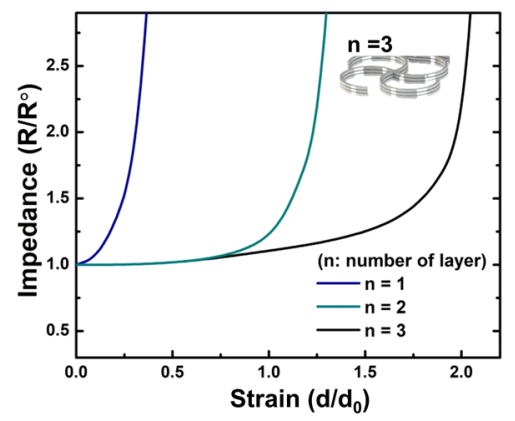

Fig. 4 3D printed dry electrocardiogram (ECG) Electrode with conductive serpentines. Schematics of a 3D printing process of serpentine electrodes on the non-auxetic origami surface, and $\mathbf{b}$ the folding and unfolding behaviors of non-auxetic origami. Gap distance, $d$, is wider once the origami is compressed. The direction of force, $f$, during the folding is highlighted with red arrows. c Relation of gap distance with height changes of $z$-direction. d Optical microscopic images of the 3D printed electrodes with the different layered number, $n$. e The different strain values from 0 to 1.8 with the sample of $n=1 \sim 3\left(R_{0}=3.46 \times 10^{-1} \Omega\right)$. $\mathbf{f}$ The normalized impedance of the 3D printed serpentine electrodes with different printing layers.

represented, and their conductivity based on the strain is tested, as shown in Fig. 4f. With $n=3$ of the printed electrodes in Fig. $4 \mathrm{~d}$ (ii), until the strain changes to 1.8 , very few bisected or disconnected conductive paths are observed. Thus, it is concluded that the printed serpentine electrodes with $n=3$ are appropriate for the ECG electrodes of the LIO sensor. In addition, the impedance of the electrodes with different $n$ values is shown in Fig. 4e. At $n=1$, the printed conductive path is disconnected at a strain of 0.15 such that the impedance is drastically increased to create an open circuit. At $n=2$, the electrodes were reliable until the strain reached 0.8. Thicker electrodes are expected to show improved conductive reliability when stretched. Once a curved object is stretched, it fails by buckling under tensile loads ${ }^{39}$. Thus, the critical buckling force was applied to the object owing to the compressive stress perpendicular to the stretching direction ${ }^{39}$. In the serpentine design, the force is applied to the middle of the curvatures in the patterns. Thus, the design of a structure requiring a higher critical buckling force $\left(\sigma_{\mathrm{cr}}\right)$ and the global tensile stress to trigger failure by buckling can enhance stretchability. The critical buckling force of the stretched plate is described by Eq. $3^{40}$

$\sigma_{\mathrm{cr}}=k E(t / w)^{2}$

where $E, t$, and $w$ are the elastic modulus, thickness, and width of the plate, respectively. $k$ represents the buckling factor, which is the ratio of length and width $(I / w)$ with a fixed Poisson's ratio. At $n=1, t / w$ is approximately 0.4 , indicating a lower $\sigma_{c r}$, resulting in easier failure through buckling than the design with $n=3(t / w=1.3)$. In addition, when $t / w>1$, the critical buckling force is predominantly affected by the elastic modulus of the materials, implying that the stretching behavior of the electrodes is converted to bending features instead of mechanical features related to buckling failure ${ }^{40}$. Thus, the electrodes with $n=3$ can be reliably conductive until they are stretched to a strain of 1.8 .

\section{ECG sensing humanoid robot with LIO}

The LIO with serpentine electrodes was developed for ECG sensors, the so-called LIO sensors. Its skin impedance has been measured. As shown in Supplementary Fig. 4, the skin impedance of electrodes with $\mathrm{LIO}$ is about $23 \%$ lower than that without $\mathrm{LIO}$ at all frequency range, indicating that there's little contact resistance attributed to LIO folding behavior. As shown in Fig. 5a, two thimble-type LIO sensors with dry ECG electrodes were prepared on the fingertip. The $\mathrm{LIO}$ sensors were integrated into the middle fingers of the humanoid robot. Figure $5 b$ shows the ECG sensing position and process of the humanoid robot, which is also presented in Supplementary Videos 3 and 4. By conformal contact with the human chest, the humanoid robot detects the ECG signals and displays the results on the tablet monitor. The summarized operating logic of the humanoid robot is shown in Supplementary Fig. 5. The voice input, 'check' is the triggering word to start the ECG sensing logic for the robot. After the detection of the word 'check', the robot first starts checking whether obstacles interrupting its arm raising are in front of the robot. The robot then performs the ECG sensing tasks if effective signals are obtained. After $30 \mathrm{~s}$ of ECG recording, the robot displays the results on the tablet monitor. Once the sensing fingers approach and push toward the chest, the LIO sensors expand and hold contact during the measurement of the ECG signals. No damaged electrodes were observed on the LIO sensors during the repeated measurements; this was attributed to the structural properties of the LIO-serpentine combination, as 

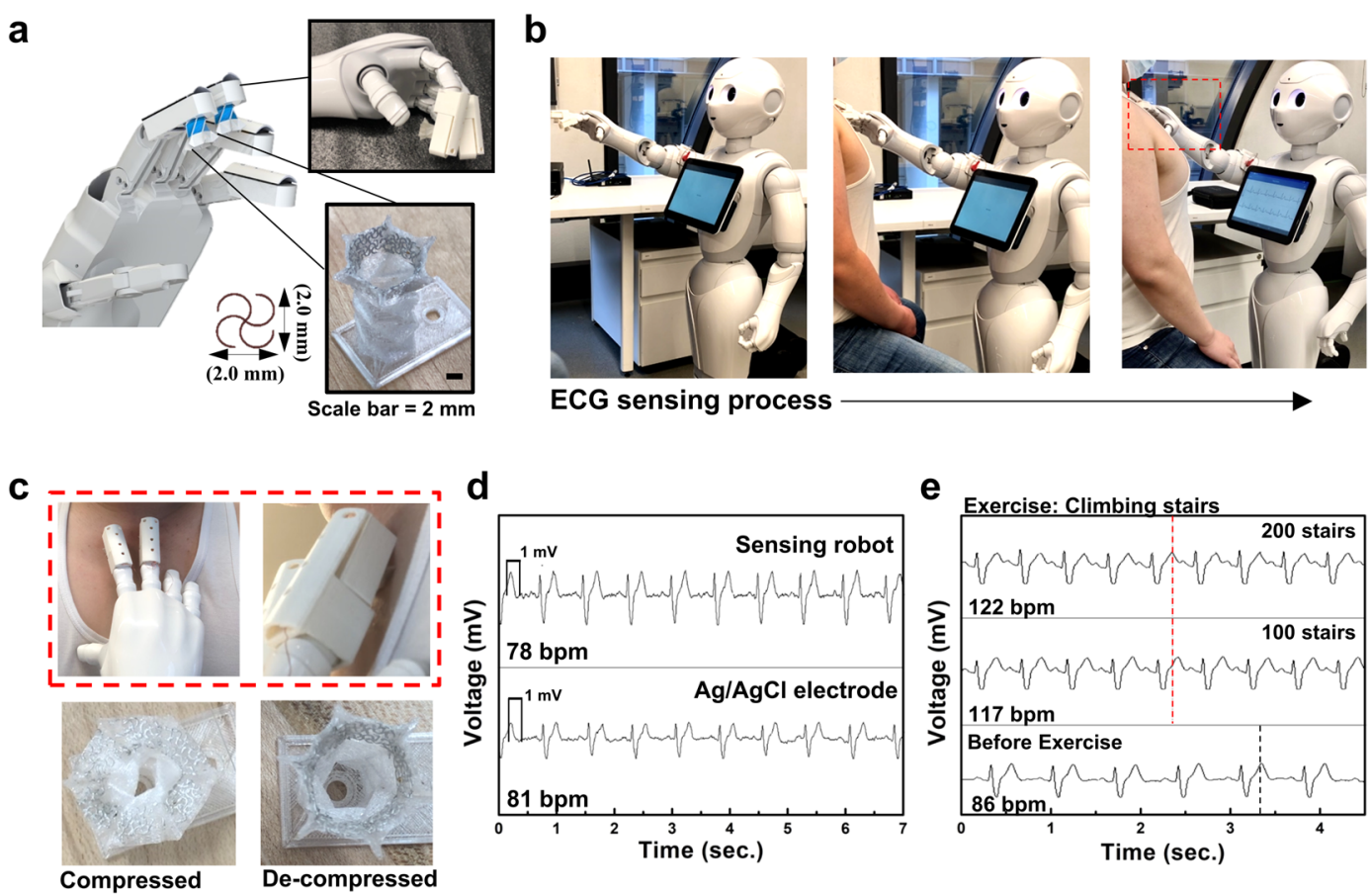

Fig. 5 Electrocardiogram (ECG) monitoring sensing robot with LIO. a Schematic of the equipped LIO sensor for ECG measurement on the humanoid robot fingertip. b The actual images of the ECG sensing process through human-robot interaction. All photos are produced under the agreement of the participant. c The detailed images of the robot fingers near the contact. The ECG signals are collected near the heart of the human subject. d Comparison of ECG signals from the sensing robot and conventional wet ECG sensor. e Collected ECG signals before and after the exercise.

shown in Fig. 5c. The measured ECG signals are presented in Fig. $5 \mathrm{~d}$, compared with the wet $(\mathrm{Ag} / \mathrm{AgCl})$ electrodes. No significant difference exists between them; the voltage range for the ECG signals was similar, and the ECG signal peaks were highly distinguishable. Moreover, the signal-to-noise ${ }^{41}$ ratio of the measured ECG with the LIO sensor and conventional wet electrodes was $21.7 \pm 0.56$ and $18.1 \pm 0.85 \mathrm{~dB}$, respectively. Thus, it is concluded that the ECG sensing ability of the humanoid sensing robot is comparable to that of conventional wet-type ECG sensors. The repeatability and lifetime ${ }^{42}$ test have been conducted as shown in Supplementary Fig. 6. Although SNR and ECG output values show slight fluctuation, their values are reliable within the error range of $20 \mathrm{~dB}$ and $0.42 \mathrm{mV}$ for the 60 sensing cycles as shown in Supplementary Fig. 6a, b, respectively. Moreover, after storing the LIO sensor in the ambient condition for 30 days, ECG output shows about $0.41 \mathrm{mV}$ as shown in Supplementary Fig. 6c. However, the ECG output is decreased with longer storing time potentially because of dust or contamination of the electrodes.

ECG monitoring with different exercise conditions is shown in Fig. 5e. Two different levels of exercise were tested by climbing 100 and 200 stairs to make the heart beat faster. Before climbing, a heartbeat rate of $86 \mathrm{bpm}$ was detected in the human subject. After climbing 100 stairs, the heartbeat rate increased to $117 \mathrm{bpm}$. After climbing, more peaks of ECG signals are detected in the same time interval of the signals before climbing, resulting in the 5 th peak at approximately 2.5 and $3.3 \mathrm{~s}$ for 117 and $86 \mathrm{bpm}$, respectively. Thus, the peak patterns of the ECG signals reflect the changes in bpm during exercise. After climbing 200 stairs, 122 $\mathrm{bpm}$ was measured from the human subject. As indicated by the red dotted line in the graph, the time for the 5th peak of its ECG signals is slightly shifted to $2.3 \mathrm{~s}$. From the results, it can be concluded that the sensing robot detects distinctive ECG signals even with slight changes in the heartbeat. After the exercise, the human body cannot hold fixed gestures because of variable breathing. In this condition, the measurement of reliable ECG signals with a dry electrode is challenging without tightening supports or holders, such as a belt. Nevertheless, the ECG sensing robot detects the signal, which is attributed to the improved contact between the skin and the LIO sensors.

\section{Blood-pressure monitoring humanoid robot}

The performance of the paired sensing robot with ECG and PPG for BP monitoring is shown in Fig. 6. The BP was monitored using the LIO-based ECG sensing hand and PPG sensing hand, as shown in Fig. 6a. The underlying principle of BP monitoring is based on the relationship between the time taken for blood to travel from the heart to the distal position ${ }^{43}$. This "time delay" is utilized as the pulse arrival time (PAT) ${ }^{44}$. The PAT is calculated from the time difference between the R-peak of the ECG and a characteristic point of the PPG peak. The ECG sensing hand was positioned at the chest of the human subject for the ECG signal near the heart, and PPG sensing hands were placed on the distal side of the subject, as shown in Fig. $6 \mathrm{~b}$ and Supplementary Video 5 . Both sensing hands are shown in Fig. $6 c$. The time difference between the R-peak and PPG peaks was obtained from the very beginning of the pulse waves, as shown in Fig. $6 \mathrm{~d}$. The relationship between $\mathrm{BP}$ and PAT is described by Eq. $4^{45}$

$\mathrm{BP}=\frac{1}{\gamma}\left[\ln \left(\frac{2 \rho D L^{2}}{a E_{0}}\right)-2 \ln T\right]$

where $T, E_{0}$, and $\gamma$ are the PAT and the coefficient of Young's modulus for the artery with and without $\mathrm{BP}$, respectively. $\rho, a, D$, and $L$ are the density of blood in the artery, the wall thickness, the diameter of the artery, and the length between the heart and the distal site. Equation 4 can be simplified to Eq. $5^{4}$

$\mathrm{BP}=\beta_{0} \times \mathrm{PAT}+\beta_{1}$

A least-squares algorithm is usually implemented to estimate the unknown coefficients $\beta_{0}$ and $\beta_{1}$, which is a calibration process ${ }^{4,7}$. 

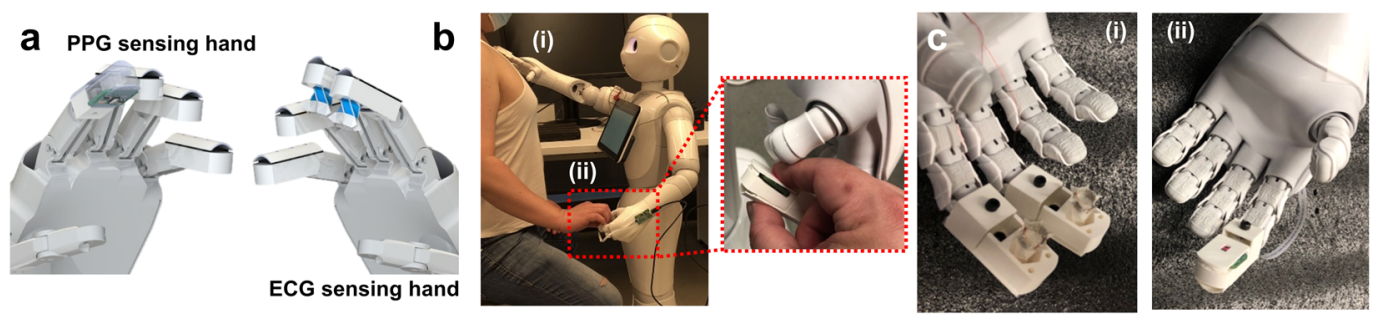

d

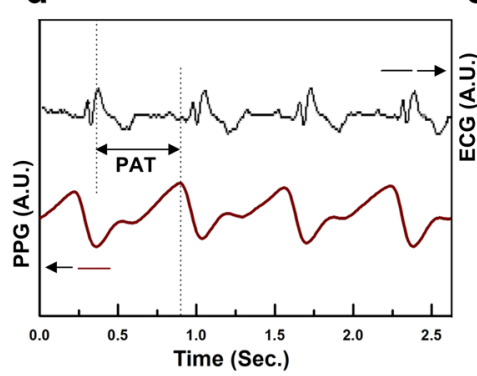

e

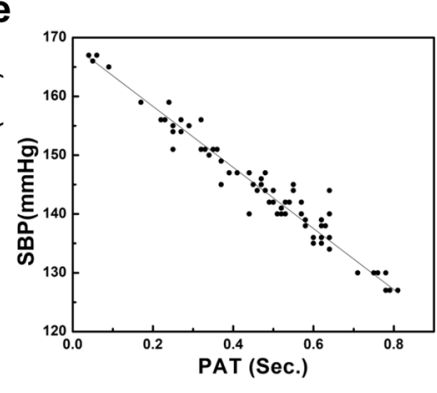

f

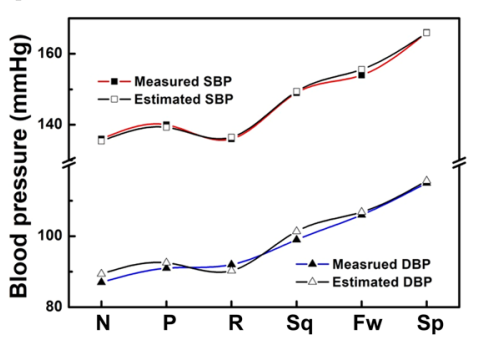

Fig. 6 Blood pressure (BP) monitoring sensing robot system. a Schematic of the robot hand equipped with PPG sensor on the left and ECG sensor on the right. $\mathbf{b}$ The actual images of the sensing process through human-robot interaction for BP measurement. All photos are produced under the agreement of the participant. c Their actual photos of ECG (i) and PPG (ii) sensing robot hands. $\mathbf{d}$ The overlaid ECG and PPG graphs to obtain pulse arrival time (PAT). e The diagrams of monitored systolic blood pressure (SBP) obtained from a sphygmomanometer with their corresponding PAT. From the diagram, the linear fit model is used to obtain the estimated SBP. $\mathbf{f}$ Comparison of measured and estimated SBP and diastolic blood pressure (DBP) at different postures or exercises (N: Normal, P: Push-up, R: rest, Sq: Squat, Fw: fast walking, and Sp: Sprint).

In the calibration process, the systolic blood pressure (SBP) and diastolic blood pressure (DBP) of the subjects were investigated using a cuff-based digital sphygmomanometer over the brachial artery of the left upper arm. Simultaneously, ECG and PPG sensing of the subject were conducted for improved time synchronization. The measured PAT at different SBP and DBP values are plotted in Fig. 6e and Supplementary Fig. 7a, respectively. The unknown coefficients of Eq. 5 were calculated from the scatterplots.

$\mathrm{SBP}=-50.23 \mathrm{PAT}+168.12$

$\mathrm{DBP}=-42.04 \mathrm{PAT}+116.42$

The coefficients of determination for Eqs. 6 and 7 were 0.948 and 0.890 , respectively. From the equations, we obtained the estimated SBP and DBP once the PAT was measured from the subject. The Bland-Altman plot ${ }^{46,47}$ was used to analyze the validity of the measured and estimated BP in Supplementary Fig. $7 b, c$. The $x$-axis of the plots indicates the average of the estimated and measured BP, and the $y$-axis represents the difference between them. The mean difference for SBP and DBP are -0.03 and $0.00 \mathrm{mmHg}$, respectively $(\leq 5 \mathrm{mmHg}$, based on the Association for the Advancement of Medical Instrumentation ${ }^{4,47}$ (AAMI) criteria). The standard deviation of 2.13 and $2.58 \mathrm{mmHg}$ are obtained for SBP and DBP, respectively ( $\leq 8 \mathrm{mmHg}$, AAMI criteria), summarized in Supplementary Table $2^{4,47}$. Thus, we concluded that the equations from the calibration process are valid for the measurement of the additional BP monitoring of the subject under different exercise conditions. However, more calibration processes must be conducted with more subjects for the universal utilization of BP monitoring. The estimated and measured BP values under different exercise conditions are presented in Fig. $6 f$. BP monitoring with four different exercises was performed. First, BP monitoring immediately after the push-up case noted as $P$ of the subject showed an increasing trend of the estimated SBP and DBP from 135 to $140 \mathrm{mmHg}$ and 87 to $91 \mathrm{mmHg}$, respectively. Simultaneously, the measured BP values from upper-arm monitoring show SBP from 136 to $139 \mathrm{mmHg}$ and DBP from 86 to $93 \mathrm{mmHg}$, respectively. Next, the BP was measured with three different exercise conditions: squat exercise (Sq), fast walk (Fw), and sprint (Sp). Drastic increases in estimated SBP and DBP after $\mathrm{Sq}$, Fw, and $\mathrm{Sp}$ ranging from 150 to $167 \mathrm{mmHg}$ and $101-116 \mathrm{mmHg}$, respectively was observed. By comparison, the measured BP with the upper-arm monitoring device showed 149-166 and 99-115 mmHg for SBP and DBP, respectively. These results imply that the $\mathrm{BP}$ estimated using the sensing robot is compatible with cuff-measured BP under different exercise conditions. Moreover, the PAT includes a pre-ejection period with pulse transit time $(\mathrm{PTT})^{3,47,48}$. The pre-ejection period is the time taken to convert electrical signals into a mechanical pumping force of the heart and isovolumetric contraction to open the aortic valves ${ }^{24,48}$. We demonstrated $\mathrm{BP}$ monitoring with strenuous exercises such as Sq, Fw, and Sp, which are significantly related to ventricular contraction and vascular function, as the reasons for using PAT instead of PTT ${ }^{49,50}$. Thus, reliable BP monitoring of the LIO sensor was possible with the sensing robot.

We developed a bio-inspired origami sensor for BP monitoring sensing robot applications. By pairing PPG with ECG sensing hands, cuff-less BP monitoring is demonstrated by the human-robot interaction of the sensing robot. BP monitoring robot systems based on PAT recording require reliable ECG signals near the heart and PPG signals at the distal site. In addition, frequent measurement with less labor required by healthcare workers is in high demand. Thus, an ECG sensor that enables stable positioning of dry ECG electrodes on the skin is essential to obtain effective ECG signals and develop a BP monitoring robot system. Based on the advantages of origami, LIO has been developed for BP monitoring robot applications. Such natureinspired origami allows the improved contact of ECG sensing dry electrodes with the skin and BP monitoring even during the outof-breath condition of the subject without belts or adhesives. This feature is achieved by the simultaneous dynamics of the auxetic and non-auxetic samples in a single monolithic structure, which is the unique advantage of origami. In addition, its mechanically tunable properties allow for additional parametric studies for optimal LIO design. Moreover, the structural properties of serpentine-shaped ECG dry electrodes complement LIO design, 
contributing to stable conductivity during conformal contact with the skin of the LIO sensor. Thus, the LIO sensors show reliable ECG sensing even under out-of-breathing conditions of the human body without additional belts or tapes after being integrated into the humanoid robot. In addition, the sensing robot with LIO was implemented for paired detection of ECG and PPG for BP monitoring under different exercise conditions.

Cuffless BP monitoring is widely used because it is more convenient for patients. In addition, the demand for home health aides has increased owing to the aging population and the prevalence of heart-related diseases. Moreover, the previous and current COVID 19 pandemics provide a lesson on the importance of the remote monitoring system for BP. Consequently, the application of sensing robots for cuffless BP monitoring is a remarkable system for remote healthcare. Beyond mere healthcare, these can be a future platform or bridge between medical personnel and remote patients. Furthermore, toward continued innovation and development as the next generation of sensors, biomedical sensing robotic systems have the potential to play an essential role in the new era of remote healthcare. Although we conclude that our BP sensing robot would have a reliable performance in defining BP values, further investigation is required to make a biomedically meaningful implementation. Beyond such an intensive view of the biomedical area, the development of a human-robot interactive BP monitoring system can be a helpful tool to facilitate intensive research on human hypertension.

\section{METHODS}

\section{Research ethical approvals}

All procedures to perform the studies of the bio-medical sensing robot have been approved by the research ethics committee at Simon Fraser University in Canada: Minimal Risk Approval 2020s0193. A male volunteer with no prior medical history of chronic cardiovascular, skin, mental health disease, or physical disability was recruited for participation in this test. The signed consents including consent of photography and videos during the test and consent to be filmed and the footage to be used for publication were obtained from the individual. According to the legal term of Canada, the confidentiality of participants is strictly respected.

\section{Preparation of origami sensors}

The fused filament fabrication (FFF) 3D printer with a direct-drive type (TID3 pro, Tenlog, Itd) is used to prepare all origami designs. The filament for the 3D printer is commercial Ninjaflex TPU85A (Fenner Drivers, Inc). The designs of origami structures are prepared using Solidworks, a 3D CAD file, and Cura slicer program (Ultimaker, Ltd.) to generate their g-codes for the $3 \mathrm{D}$ printing process. The nozzle size of the hot end is $0.2 \mathrm{~mm}$. The print speed, infill, layer height, temperature, and width are $30 \mathrm{~mm} \mathrm{~s}^{-1}, 0.1 \mathrm{~mm}$, $223^{\circ} \mathrm{C}$, and $0.1 \mathrm{~mm}$, respectively. The commercial co-polyester filament (ColorFabb, Itd.) is used for the sensor frames, and the FFF 3D printer with the bowden tube type (Ultimaker 3) is utilized to print. The g-code preparation is the same as above.

\section{Characterization of the 3D printed origami sensor}

Mechanical properties of the origami designs were investigated using a universal testing machine, Shimadzu EZ-LX. The compression and pull-off tests were conducted at a loading rate of $2 \mathrm{~mm} \mathrm{~min}^{-1}$. For the parametric studies of the origami design, the loading force is applied by using a universal testing machine, and all of the necessary information such as length, width, height, and thickness are measured by using a vernier caliper very carefully. For the pumping behavior of the origami design, as shown in Supplementary Video 2 and Fig. 1f, the small hole of the origami design on the bottom is fully sealed by using commercial silicone rubber (Eco-flex 00-30, Smooth-on, Inc.).

\section{Preparation and characterization of serpentine electrodes}

The conductive silver paste for serpentine electrodes was prepared by dissolving $0.4 \mathrm{~g}$ polyurethane into a blend $(1 \mathrm{ml} \mathrm{N}, \mathrm{N}$-dimethylformamide (DMF) with $4 \mathrm{ml}$ tetrahydrofuran (THF)) by SpeedMixer DAC150.1 FVZ-K
(FlackTek, Inc.), followed by adding $2 \mathrm{~g}$ silver flake (Inframat Advanced Materials, US). After thoroughly mixing, the viscosity of the silver paste is controlled by heating at $65^{\circ} \mathrm{C}$ for the ambient time. Then, serpentine electrodes are programmed and fabricated by a direct writing printer SHOT mini 100Sx (Musashi Engineering, Inc.) with $1 \mathrm{~mm} \mathrm{~s}^{-1}$, followed by cured at $80^{\circ} \mathrm{C}$ for $1 \mathrm{~h}$. The stretching behavior of the serpentine electrodes is monitored by using an optical microscope (OT-HD, Opti-tekscope, Itd). For the impedance of the electrodes, the electrodes are printed on the flexible plate prepared by using an FFF 3D printer. A pair of the conductive path is connected to digital multimeters and monitored the impedance changes as a function of stretched distance. Also, the skin impedance of dry serpentine electrodes with and without $\mathrm{LIO}$ was measured using an LCR meter (Agilent, E4980A, Itd). The measurement was conducted in the frequency range from $20 \mathrm{~Hz}$ to $400 \mathrm{kHz}$.

\section{ECG monitoring}

The LIO sensors are equipped to the two fingers of the humanoid robot. The logic for the programming is represented in Supplementary Fig. 5. The ECG sensors are connected to a commercial ECG sensing system (Pulsebit EX, Wellue Ltd.). Also, an entire ECG system is integrated onto Softbank's Pepper humanoid robot. The duration to record the ECG signals of the human subject is $30 \mathrm{~s}$ for one trial. The ECG sensing system has $\pm 3 \mathrm{mV}$ of the input dynamic range and $0.05-40 \mathrm{~Hz}$ bandwidth. After measuring the ECG signals by the system, the recorded signals are automatically transferred to the robot via wireless Bluetooth. The prepared LIO sensor, a LIO design with dry serpentine electrodes, is used for the humanoid robot to measure the ECG signals. For the comparison, the conventional wet $(\mathrm{Ag} / \mathrm{AgCl})$ electrodes with an ECG sensor are used with the same sensing system at the same position.

\section{Blood pressure monitoring}

A commercial PPG sensor (MAX30102, Maxim integrated, Ltd.) is paired with the developed LiO sensor to integrate onto Softbank's Pepper humanoid robot. The LIO sensor fingertip on the sensing hand (left hand) of the robot is positioned on the subject's chest, and the PPG sensing hand (right hand) is touching the subject's fingertip. The waveforms obtained from ECG and PPG sensing hands are collected simultaneously for time synchronization. At the same time, a cuff-type digital sphygmomanometer is employed to obtain systolic and diastolic BP for reference from the left upper arm. For the BP measurement from ECG-PPG sensing hands, the calibration process of BP for the subject is carried out with the linear square model in Supplementary Table 1. The PAT is calculated based on the time difference between two R-wave and systolic wave peaks from ECG and PPG signals.

\section{Ethics information for ECG and BP monitoring}

A 36-year-old male student is employed as the human subject for ECG and $\mathrm{BP}$ monitoring. His height and weight are $178 \mathrm{~cm}$ and $88 \mathrm{~kg}$, respectively. Dry LIO sensors of the humanoid's fingertip are positioned on his chest for ECG measurement. The subject has performed two different exercises climbing 100 and 200 stairs for the ECG measurement. The subject has carried out four different exercises: 20 times of push-up, rest for $20 \mathrm{~min}, 30$ times of squat, fast walking, and a sprint of $50 \mathrm{~m}$ for the BP monitoring with varied heart rates. All tests have been performed after getting written permission from the subject and the approval of research ethics. All Supplementary Videos are produced under the agreement of the participant.

\section{DATA AVAILABILITY}

All relevant data that support the findings of this study are available from the corresponding author upon reasonable request.

Received: 29 September 2021; Accepted: 21 December 2021; Published online: 27 January 2022

\section{REFERENCES}

1. Chang, D., Xu, H., Rebaza, A., Sharma, L. \& Cruz, C. S. D. Protecting health-care workers from subclinical coronavirus infection. Lancet Respir. Med. 8, E13 (2020).

2. Ates, H. C., Yetisen, A. K., Güder, F. \& Dicer, C. Wearable devices for the detection of COVID-19. Nat. Electron. 4, 13-14 (2021). 
3. $\mathrm{Xu}, \mathrm{H}$. et al. Flexible organic/Inorganic hybrid near-infrared photoplethysmogram sensor for cardiovascular monitoring. Adv. Mater. 29, 1700973 (2017).

4. Elgendi, M. et al. The use of photoplethysmography for assessing hypertension. Npj. Digit. Med. 2, 60 (2019).

5. Ha, T. et al. A chest-laminated ultrathin and stretchable E-Tattoo for the measurement of electrocardiogram, seismocardiogram, and cardiac time intervals. Adv. Sci. 6, 1900290 (2019).

6. Park, S. et al. Ultraflexible near-Infrared organic photodetectors for conformal photoplethysmogram sensors. Adv. Mater. 30, 1802359 (2018).

7. Sharma, M. et al. Cuff-less and continuous blood pressure monitoring: A methodological review. Technologies 5, 21 (2017).

8. Liang, Y. et al. How effective is pulse arrival time for evaluating blood pressure? challenges and recommendations from a study using the MIMIC database. J. Clin. Med. 8, 337 (2019).

9. Park, Y.-S. et al. Real-time monitoring of blood pressure using digitalized pulse arrival time calculation technology for prompt detection of sudden hypertensive episodes during laryngeal microsurgery: Retrospective observational study. J. Med. Internet Res. 5, e13156 (2020).

10. Ebrahim, M. P. et al. Blood pressure estimation using on-body continuous wave radar and photoplethysmogram in various posture and exercise conditions. Sci. Rep. 9, 16346 (2019).

11. $\mathrm{Wu}, \mathrm{H}$. et al. Materials, devices, and systems of on-skin electrodes for electrophysiological monitoring and human-machine interfaces. Adv. Sci. 8, 2001938 (2021).

12. Searle, A. \& Kirkup, L. A direct comparison of wet, dry, and insulating bioelectric recording electrodes. Physiol. Meas. 21, 271-283 (2000).

13. Kaur, M., Kim, T.-H. \& Kim, W. S. New frontiers in 3D structural sensing robots. Adv. Mater. 33, 2002534 (2021).

14. Chi, M., Zhao, J., Dong, Y. \& Wang, X. Flexible carbon nanotube-based polymer electrode for long-term electrocardiographic recording. Materials 12, 971 (2019).

15. Fu, Y., Zhao, J., Dong, Y. \& Wang, X. Dry electrodes for human bioelectrical signal monitoring. Sensors 20, 3651 (2020).

16. Choi, S. B. et al. Conformable, thin, and dry electrode for electrocardiography using composite of silver nanowires and polyvinyl butyral. Electron. Mater. Lett. 15, 267-277 (2019).

17. Wang, K. et al. Stretchable dry electrodes with concentric ring geometry for enhancing spatial resolution in electrophysiology. Adv. Healthc. Mater. 6, 1700552 (2017).

18. Lee, S. M. et al. Self-adhesive epidermal carbon nanotube electronics for tetherfree long-term continuous recording of biosignals. Sci. Rep. 4, srep06074 (2014).

19. Zhang, L. et al. Fully organic compliant dry electrodes self-adhesive to skin for long-term motion-robust epidermal biopotential monitoring. Nat. Commun. 11 4683 (2020).

20. Kim, T., Park, J., Sohn, J., Cho, D. \& Jeon, S. Bioinspired, highly stretchable, and conductive dry adhesives based on 1D-2D hybrid carbon nanocomposites for allin-one ECG electrodes. ACS Nano 10, 4770-4778 (2016).

21. Greiner, C., Campo, A. D. \& Arzt, E. Adhesion of bioinspired micropatterned surfaces: Effects of pillar radius, aspect ratio, and preload. Langumir 23, 3495-3052 (2007).

22. Stauffer, F. et al. Skin conformal polymer electrodes for clinical ECG and EEG recordings. Adv. Healthc. Mater. 7, 1700994 (2018).

23. Luo, N. et al. Flexible piezoresistive sensor patch enabling ultralow power cuffless blood pressure measurement. Adv. Funct. Mater. 26, 1178-1187 (2016).

24. Kim, J. \& Kim, W. S. A paired stretchable printed sensor system for ambulatory blood pressure monitoring. Sens. Actuators A: Phys. 238, 329-336 (2016).

25. Li, S., Fang, H., Sadeghi, S., Bhovad, P. \& Wang, K.-W. Architected origami materials: How folding creates sophisticated mechanical properties. Adv. Mater. 31, 1805282 (2019)

26. Zhai, Z., Wang, Y. \& Jiang, H. Origami-inspired, on-demand deployable and collapsible mechanical metamaterials with tunable stiffness. Proc. Natl Acad. Sci. USA 115, 2032-2037 (2018).

27. Kim, T.-H., Vanloo, J. \& Kim, W. S. 3D origami sensing robots for cooperative healthcare monitoring. Adv. Mater. Technol. 6, 2000938 (2021).

28. Ning, $X$. et al. Assembly of advanced materials into 3D functional structures by methods inspired by origami and kirigami: A review. Adv. Mater. Interface $\mathbf{5}$, 1800284 (2018)

29. Li, S. et al. "A Vacuum-driven Origami "Magic-ball" Soft Gripper". In 2019 International Conference on Robotics and Automation (ICRA) 7401-7408 (IEEE, 2019).

30. Baek, S.-M., Yim, S., Chae, S.-H., Lee, D.-Y. \& Cho, K.-J. Ladybird beetle-inspired compliant origami. Sci. Robot. 5, eaaz6262 (2020).

31. Kim, W. et al. Bioinspired dual-morphing stretchable origami. Sci. Robot. 4 eaay3493 (2019).

32. Feng, H., Chai, N. \& Dong, W. Experimental investigation on the morphology and adhesion mechanism of leech posterior suckers. PLoS One 10, e0140776 (2015).
33. Kampowski, T., Eberhard, L., Gallenmüller, F., Speck, T. \& Poppinga, S. Functional morphology of suction discs and attachment performance of the Mediterranean medicinal leech (Hirudo verbana Carena). J. R. Soc. Interface 13, 20160096 (2016).

34. Kampowski, T., Thiemann, L.-L., Kürner, L., Speck, T. \& Poppinga, S. Exploring the attachment of the Mediterranean medicinal leech (Hirudo verbana) to porous substrates. J. R. Soc. Interface 17, 20200300 (2020).

35. Evans, K. E. \& Alderson, A. Auxetic materials: Functional materials and structures from lateral thinking! Adv. Mater. 12, 617-628 (2000).

36. Ren, X., Das, R., Tran, P., Ngo, T. D. \& Xie, M. Y. Auxetic metamaterials and structures: A review. Smart. Mater. Struct. 27, 023001 (2018).

37. Westra, K., Dunne, F., Kulsa, S., Hunt, M. \& Leachman, J. Compliant polymer origami bellows in cryogenics. Cryogenics 114, 103226 (2021).

38. Tao, R. et al. 4D printed origami metamaterials with tunable compression twist behavior and stress-strain curves. Compos. B Eng. 201, 108344 (2020).

39. Zaccaria, D., Bigoni, D., Noselli, G. \& Misseroni, D. Structures buckling under tensile dead load. P. R. Soc. A-Math. Phys. 467, 1686-1700 (2011).

40. Rammerstorfer, F. G. Buckling of elastic structures under tensile loads. Acta Mech. 229, 881-900 (2018).

41. Bindu, H. C. H. Computational Intelligence and Big Data Analytics: Applications in Bioinformatics 81-95 (Springer, 2019).

42. Yamamoto, Y. et al. Efficient skin temperature sensor and stable gel-less sticky ECG sensor for a wearable flexible healthcare path. Adv. Healthc. Mater. 6, 1700495 (2017).

43. Wagenseil, J. E. \& Mecham, R. P. Elastin in large artery stiffness and hypertension J. Cardiovasc. Transl. Res. 5, 264-273 (2012).

44. Zheng, Y., Poon, C. C. Y., Yan, B. P. \& Lau, J. Y. W. Pulse arrival time based cuff-less and $24-\mathrm{H}$ wearable blood pressure monitoring and its diagnostic value in hypertension. J. Med. Syst. 40, 195 (2016).

45. Chen, W., Kobayashi, T., Ichikawa, S., Takeuchi, Y., \& Togawa, T. Continuous estimation of systolic blood pressure using the pulse arrival time and intermittent calibration. Med. Biol. Eng. Comput. 38, 569-574 (2000).

46. Patzak, A., Mendoza, Y., Gesche, H. \& Konermann, M. Continuous blood pressure measurement using the pulse transit time: Comparison to intra-arterial measurement. Blood Press 24, 217-221 (2015).

47. White, W. B. et al. National standard for measurement of resting and ambulatory blood pressures with automated sphygmomanometers. Hypertension 21 504-509 (1993)

48. Feng, M. et al. Validation of volume-pressure recording tail-cuff blood pressure measurements. Am. J. Hypertens. 21, 1288-1291 (2008).

49. Marie, G. V., Lo, C. R., Jones, J. V. \& Johnston, D. W. The relationship between arterial blood pressure and pulse transit time during dynamic and static exercise. Psychophysiology 21, 521-527 (1984).

50. Noordergraaf, A. Circulatory System Dynamics 1st edn (Academic, 1978).

\section{ACKNOWLEDGEMENTS}

This research was supported by the Discovery and Discovery Accelerator Supplement Grant 2016-04334, funded by the Natural Sciences and Engineering Research Council of Canada (NSERC).

\section{AUTHOR CONTRIBUTIONS}

W.S.K. created the concept of sensing robots and supervised the project. T.-H.K. designed the architected sensing robots and conducted the experiments with help from C.B. and Z.C. T.-H.K. analyzed and interpreted the data. All authors wrote and revised the manuscript.

\section{COMPETING INTERESTS}

The authors declare no competing interests.

\section{ADDITIONAL INFORMATION}

Supplementary information The online version contains supplementary material available at https://doi.org/10.1038/s41528-022-00139-x.

Correspondence and requests for materials should be addressed to Woo Soo Kim.

Reprints and permission information is available at http://www.nature.com/ reprints

Publisher's note Springer Nature remains neutral with regard to jurisdictional claims in published maps and institutional affiliations. 
(c) Open Access This article is licensed under a Creative Commons Attribution 4.0 International License, which permits use, sharing, adaptation, distribution and reproduction in any medium or format, as long as you give appropriate credit to the original author(s) and the source, provide a link to the Creative Commons license, and indicate if changes were made. The images or other third party material in this article are included in the article's Creative Commons license, unless indicated otherwise in a credit line to the material. If material is not included in the article's Creative Commons license and your intended use is not permitted by statutory regulation or exceeds the permitted use, you will need to obtain permission directly from the copyright holder. To view a copy of this license, visit http://creativecommons. org/licenses/by/4.0/.

(c) The Author(s) 2022 